\title{
Neurons derived from transplanted neural stem cells restore disrupted neuronal circuitry in a mouse model of spinal cord injury
}

\author{
Masahiko Abematsu, 1,2 Keita Tsujimura, ${ }^{1}$ Mariko Yamano, ${ }^{3}$ Michiko Saito, ${ }^{4}$ Kenji Kohno,, \\ Jun Kohyama, ${ }^{1}$ Masakazu Namihira, ${ }^{1}$ Setsuro Komiya, ${ }^{2}$ and Kinichi Nakashima ${ }^{1}$

\begin{abstract}
${ }^{1}$ Laboratory of Molecular Neuroscience, Graduate School of Biological Sciences, Nara Institute of Science and Technology, Ikoma, Japan. 2Department of Orthopaedic Surgery, Graduate School of Medical and Dental Sciences, Kagoshima University, Kagoshima, Japan. ${ }^{3}$ Department of Comprehensive Rehabilitation, Osaka Prefecture University, Habikino, Japan. ${ }^{4}$ Laboratory of Molecular and Cell Genetics, Graduate School of Biological Sciences, Nara Institute of Science and Technology, Ikoma, Japan.
\end{abstract}

\begin{abstract}
The body's capacity to restore damaged neural networks in the injured CNS is severely limited. Although various treatment regimens can partially alleviate spinal cord injury (SCI), the mechanisms responsible for symptomatic improvement remain elusive. Here, using a mouse model of SCI, we have shown that transplantation of neural stem cells (NSCs) together with administration of valproic acid (VPA), a known antiepileptic and histone deacetylase inhibitor, dramatically enhanced the restoration of hind limb function. VPA treatment promoted the differentiation of transplanted NSCs into neurons rather than glial cells. Transsynaptic anterograde corticospinal tract tracing revealed that transplant-derived neurons reconstructed broken neuronal circuits, and electron microscopic analysis revealed that the transplant-derived neurons both received and sent synaptic connections to endogenous neurons. Ablation of the transplanted cells abolished the recovery of hind limb motor function, confirming that NSC transplantation directly contributed to restored motor function. These findings raise the possibility that epigenetic status in transplanted NSCs can be manipulated to provide effective treatment for SCI.
\end{abstract}

\section{Introduction}

Numerous studies (1-18) have indicated that a variety of treatments, such as administration of antibodies against myelin-associated neurite growth inhibitors $(4,5,16)$ and neurotrophic factors $(12,13)$, can yield limited restorative benefits for spinal cord injury (SCI), but the mechanistic basis of symptomatic improvement is far from clear. Because of their ability to self renew, to differentiate into multiple lineages, and to migrate toward damaged sites, neural stem cells (NSCs) are currently considered to be promising components of cell-replacement strategies aimed at treating CNS injuries $(7-11,14,15)$.

Two desirable objectives of cell-based therapeutics are to induce trophic reactions, such as the production of extracellular matrix and diffusible factors, and to replace cells lost through injury or disease with transplant-derived cells, such as new oligodendrocytes and neurons, that will enhance the regenerative responses of the host CNS. Trophic factors secreted from transplanted cells have been shown to support neuronal survival and neurite outgrowth $(12,13)$, while other studies report that transplanted oligodendrocyte precursor cells enhance remyelination of remaining neuronal axons, leading to restoration of locomotion after SCI $(9,15)$. Stem cell-derived neuronal supplementation in the injured spinal cord also induces partial recovery, although a direct contribution of such neurons to the reconstruction of disrupted neuronal circuits has not been demonstrated $(7,10,11)$.

In the severest CNS injuries, many neurons sustain direct damage and disrupted neuronal circuits have to be restored. Inflammatory cytokines are upregulated at lesion sites in the

Conflict of interest: The authors have declared that no conflict of interest exists. Citation for this article: J Clin Invest. 2010;120(9):3255-3266. doi:10.1172/JCI42957.
CNS (19-21), but their effect is to promote astrocytic differentiation. Moreover, while exogenous NSCs that are transplanted into the injured CNS undergo proliferation, the vast majority of newly generated cells differentiate into astrocytes $(7,21,22)$. This strong bias toward astrocytic differentiation at the expense of neuronal differentiation is one of the major current obstacles in regeneration therapy.

Valproic acid (VPA; 2-propylpentanoic acid) is an established drug in the long-term treatment of epilepsy (23). Recent experiments have further revealed that VPA can directly inhibit histone deacetylase (HDAC) activity and cause hyperacetylation of histones in 293T, Neuro2A, and teratocarcinoma F9 cells $(24,25)$, and we have also found that VPA induces neuronal differentiation but suppresses astrocytic and oligodendrocytic differentiation of NSCs (26). Taking advantage of these newly discovered effects of VPA, we report here that functional recovery in SCI model mice is dramatically improved by a combined treatment involving NSC transplantation and VPA administration. Furthermore, we reveal the precise mechanisms used to reconstruct the damaged corticospinal tract (CST); these differ from neurite regrowth and remyelination of host neurons, hitherto considered as the major reasons for functional recovery of SCI following NSC transplantation.

\section{Results}

Combined NSC transplantation and VPA administration improves functional recovery of hind limbs without CST axon reextension. As VPA has been shown to have effects that are likely to be beneficial to treatment of the injured CNS, such as neuroprotection (27-31), induction of neuronal differentiation (26), and promotion of neurite outgrowth (32), we examined the response of SCI model 
A
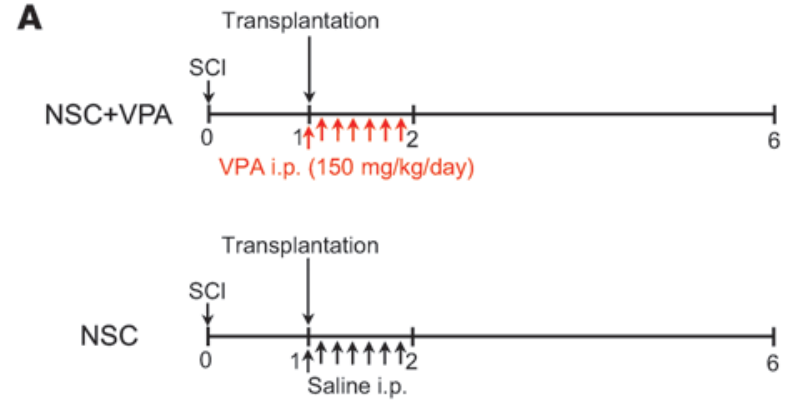

VPA
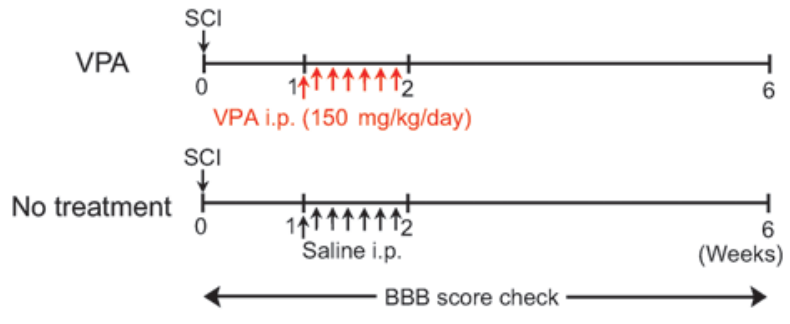

C
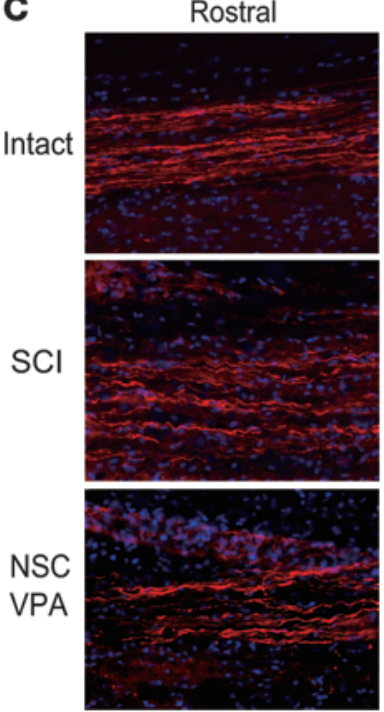

B

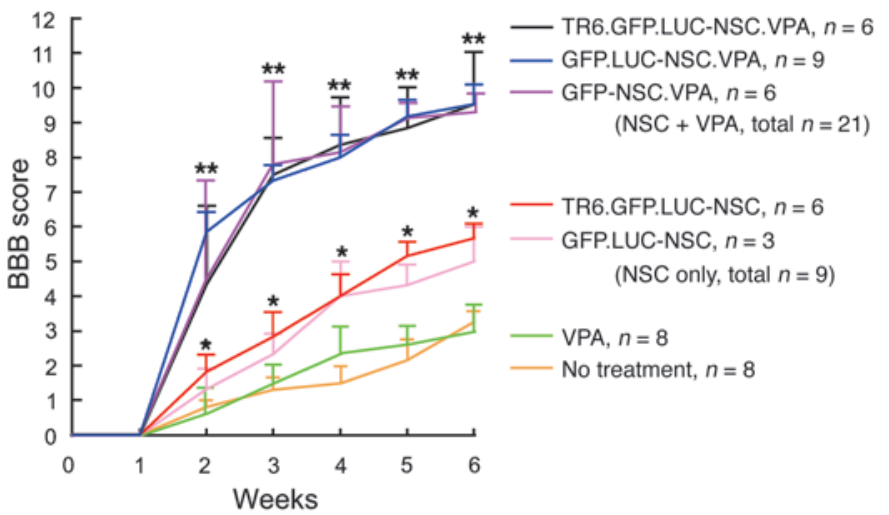

D

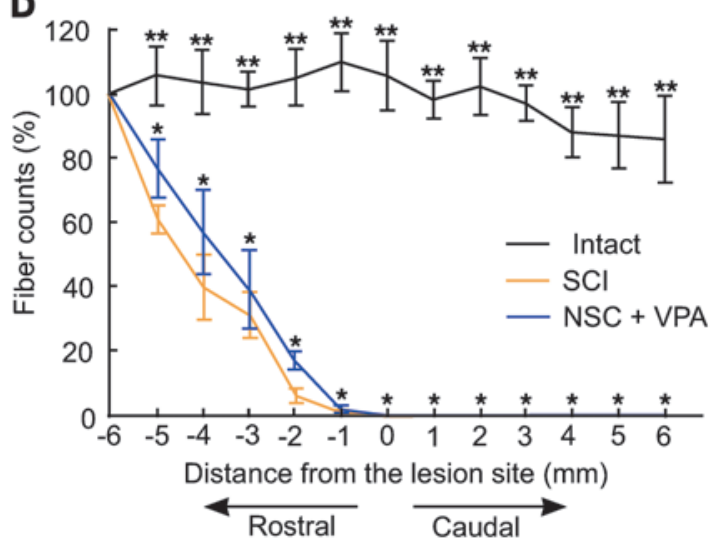

Figure 1

A combination of NSC transplantation and VPA administration improves functional recovery of hind limbs without CST axon reextension. (A) Schematic of the NSC transplantation and VPA injection protocol. (B) Time course of functional recovery of hind limbs after SCI. GFP-NSCs, GFP.LUC-NSCs, and TR6.GFP.LUC-NSCs were transplanted into the SCl epicenter 7 days after injury as indicated. Combined treatment with NSC transplantation and VPA administration resulted in the greatest functional recovery. Data represent mean \pm SEM. ${ }^{\star \star} P<0.001$ compared with $\mathrm{SCl}$ models with no treatment; ${ }^{*} P<0.01$ compared with $\mathrm{SCl}$ models with no treatment (repeated measures ANOVA). NSC+VPA, total $n=21$. (C) Representative pictures of BDA-labeled CST fibers at $5 \mathrm{~mm}$ rostral and $5 \mathrm{~mm}$ caudal to the lesion site. BDA was injected into the motor cortices 12 weeks after SCl. 2 weeks after the injection, mice were fixed and spinal cord sections were stained. Representative results for a GFP-NSC-transplanted spinal cord are shown. Blue, Hoechst nuclear staining. Scale bar: 20 um. (D) Quantification of the labeled CST fibers in the spinal cords of intact mice, $\mathrm{SCI}$ mice receiving no treatment, and SCI mice undergoing combined NSC/VPA treatment. Eight 30- $\mu$ m-thick serial parasagittal sections from individual spinal cords were evaluated. The $x$ axis indicates specific locations along the rostrocaudal axis of the spinal cord, and the $y$ axis indicates the ratio of the number of BDA-labeled fibers at the indicated site to that at $6 \mathrm{~mm}$ rostral to the lesion site (Th9). ${ }^{*} P<0.001$ compared with SCI models without treatment; ${ }^{*} P=0.188$ There is no significant difference in the number of BDA-labeled fibers between NSC+VPA-treated mice (blue line) and SCI model mice with no treatment (yellow line) (repeated measures ANOVA). All data shown are from at least 3 experiments in parallel conditions, with error bars representing SEM.

mice to different combinations of VPA administration and NSC transplantation. We prepared NSCs from embryonic forebrains of 3 different $\mathrm{Tg}$ mouse lines ubiquitously expressing either GFP (GFP-Tg) (33), GFP and LUC (GFP.LUC-Tg), or GFP, LUC, and the diphtheria toxin (DT) receptor human heparin-binding EGF-like growth factor (TR6) (TR6.GFP.LUC-Tg) (see Methods). The expres- sion of GFP, LUC, and TR6 in NSCs enabled us to distinguish transplanted cells from host cells, to trace the survival of transplanted cells based on LUC activity in a noninvasive fashion, and to specifically ablate transplanted cells (see below), respectively. To obtain a homogeneous population of NSCs, we used adherent monolayer culture (34-36). The embryonic forebrains were 
A
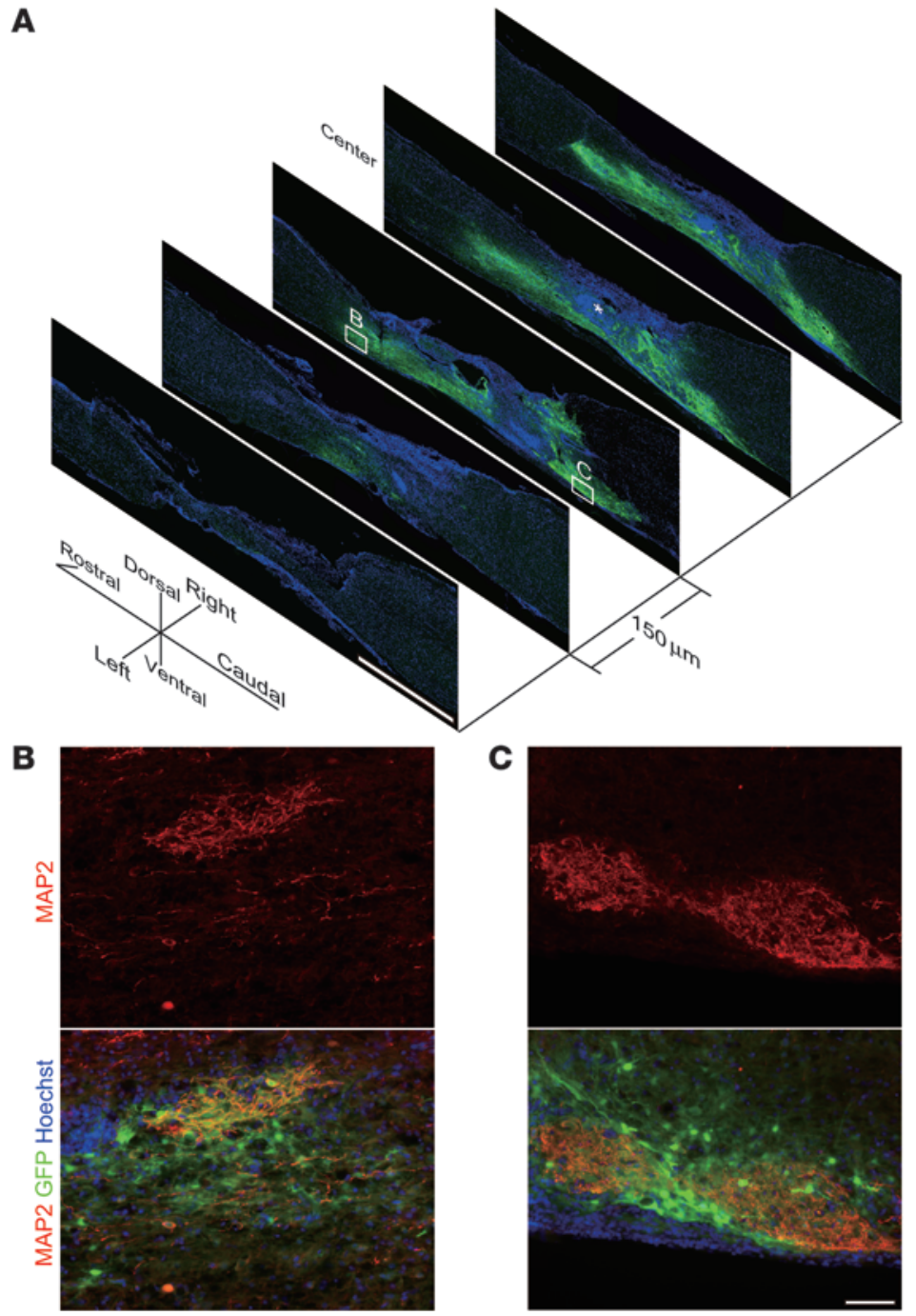

C

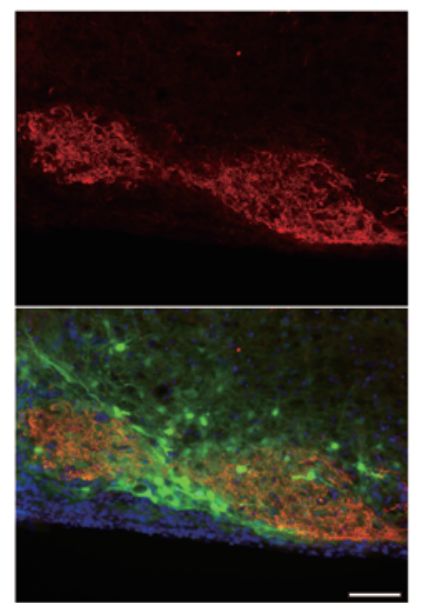

Figure 2

Transplanted NSCs migrate from the injection site and encompass the lesion site. Representative results of GFP-NSC-transplanted SCI model mice are shown. (A) A series of immunostaining images of injured spinal cord at 6 weeks after injury. SCI mice received combination treatment with NSC transplantation and VPA administration. Specimens were picked up every $150 \mu \mathrm{m}$ and stained with anti-GFP (green) and MAP2 (not shown) antibodies and Hoechst (blue). The epicenter of the $\mathrm{SCl}$ is indicated $\left(^{*}\right)$. Scale bar: $1 \mathrm{~mm}$. (B and C) Higher-magnification images of the white boxes in A. GFP-positive transplanted NSCs differentiated into MAP2-positive neurons and extended their processes. Scale bar: $50 \mu \mathrm{m}$.

dissociated and cultured with EGF and basic FGF (bFGF) (36) (Supplemental Figure 1, A and B; supplemental material available online with this article; doi:10.1172/JCI42957DS1). These cells uniformly expressed the stem cell markers Sox 2 and nestin but did not express differentiation markers (Supplemental Figure 1, $\mathrm{C}$ and $\mathrm{D}$ ). Under the appropriate conditions for each lineage, these NSCs differentiated into neurons, astrocytes, or oligodendrocytes (Supplemental Figure 1, E and F). NSCs from different Tg mice behaved similarly in these culture conditions (data not shown). NSCs that had been cultured and passaged 5-10 times in the presence of both EGF and bFGF to maintain the undifferentiated state were used for transplantation studies.
Undifferentiated NSCs were transplanted into the SCI epicenter 7 days after injury. Nontransplanted control and transplanted mice were then intraperitoneally administered VPA or saline daily for 7 days (Figure 1A), whereafter we monitored their hind limb motor function using the open field locomotor scale (BBB score) $(7-9,37)$ for 6 weeks. Remarkably, we found that the simultaneous treatment of SCI model mice with NSCs and VPA resulted in a dramatic recovery of hind limb function compared with either treatment alone (Figure 1B and Supplemental Videos 1-4). There were no significant differences among the data obtained from each SCI model mouse group transplanted with the 3 distinct NSCs. Functional recovery of each treated SCI model mouse reached a plateau at around 6 weeks, the level of which was sustained for more than 3 months. Since mice treated with VPA alone showed no further improvement compared with untreated mice, it is most likely that VPA affected the function of transplanted cells.

We next sought to determine the basis for this improvement in locomotor function. Since transplanted NSCs have been reported to play a supportive role in the reextension of injured axons (14), we analyzed whether CST axons were regenerated by anterograde labeling using biotinylated dextran amine (BDA) $(6,16,17)$. Because BDA was injected into the motor cortex, only the axons of first-order neurons in the CST could be visualized (Figure 1C). In our SCI model mice, the caudal part of the injured site was completely devoid of CST axons (Figure 1, C and D), and the same was true in mice that had undergone combined NSC transplantation and VPA administration (Figure 1, C and D). These data indicated that CST axons did not reextend in mice treated with both NSCs and VPA and therefore that some other mechanism was responsible for the animals' dramatic functional locomotor improvement.

Transplanted NSCs encompass the lesion site and extend their processes. Given that host CST axon reextension was not involved in the observed hind limb recovery, we decided to focus on the transplanted cells. We analyzed the migration, morphology, neuronal marker expression, and viability of these cells after coadministration with VPA. Transplant-derived cells migrated to both rostral and caudal areas and displayed processes that extended into the gray matter and dorsal funiculus within 5 weeks of transplantation (Figure 2). Between $20 \%$ and $40 \%$ of the transplanted cells were found to be surviving in the injured spinal cord after 8 weeks, and 17\% still remained viable more than 1 year after transplantation (data not shown). About $20 \%$ of the surviving cells had differentiated into microtubule-associated protein 2-positive (MAP2-positive) neurons with elongated processes within 5 weeks after transplantation (Figure 2, B and C, and Figure 3, E and F). Survival of the transplanted NSCs was not significantly influenced by VPA administration (Supplemental Figure 8).

HDAC inhibition promotes neuronal differentiation of NSCs and is critical for transplantation-induced hind limb recovery. In contrast to previous studies, which have indicated that very few transplanted NSCs differentiate into neurons in the injured CNS environment $(8,10,11$, $20)$, many neurons were observed in the spinal cord after coadministration with VPA. We next examined in more detail the contribution of VPA to differentiation of cultured and transplanted NSCs. To analyze differentiation in vitro, NSCs were treated with either 

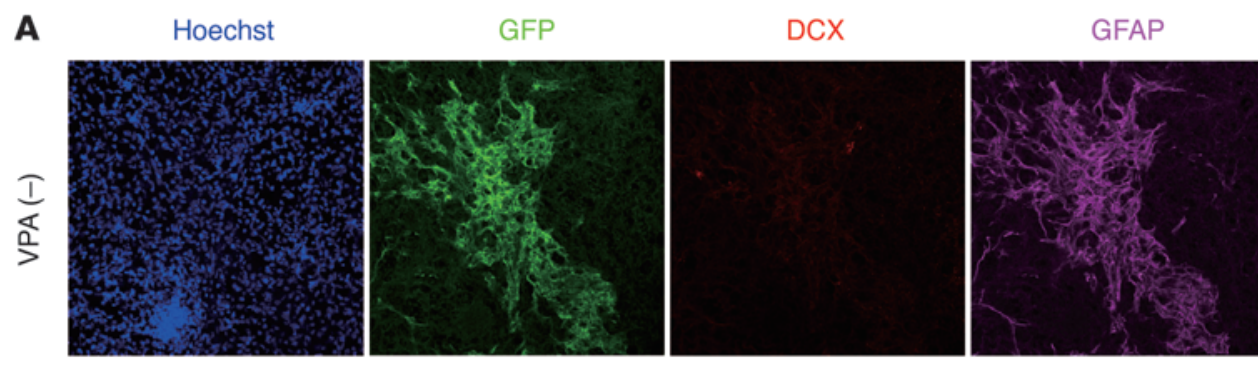

Hoechst/GFP/DCX/GFAP

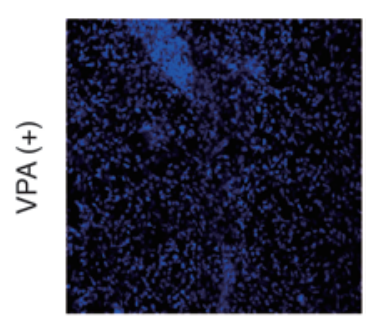

B

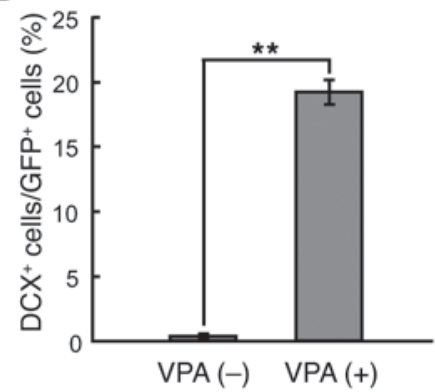

E
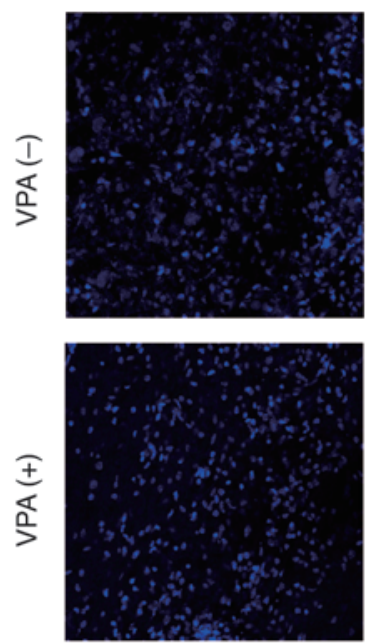

$\mathbf{F}$

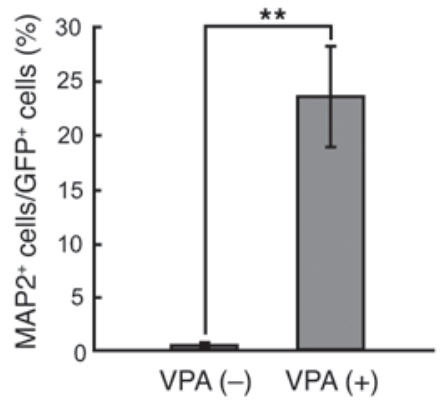

GFP
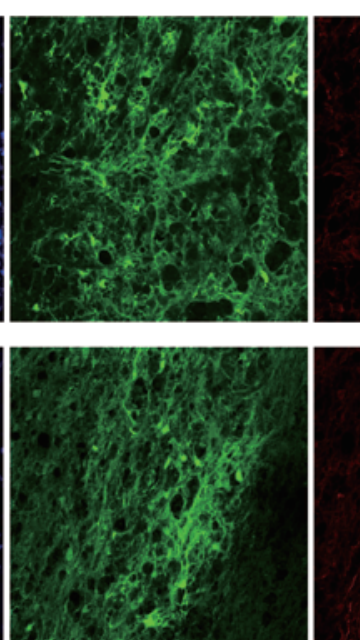

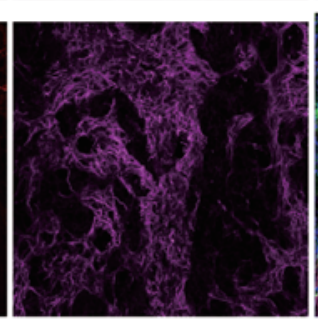

C

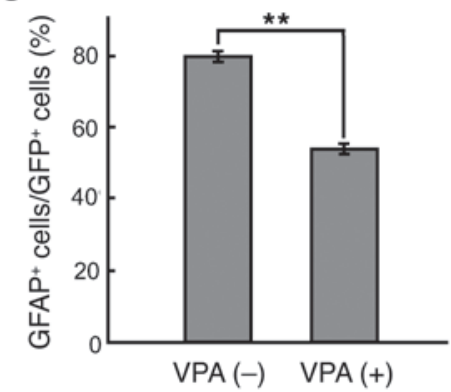

MAP2

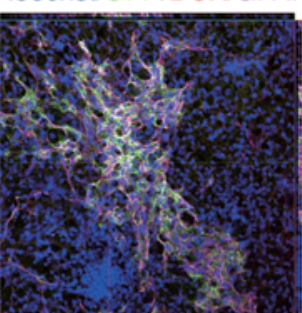

Caudal

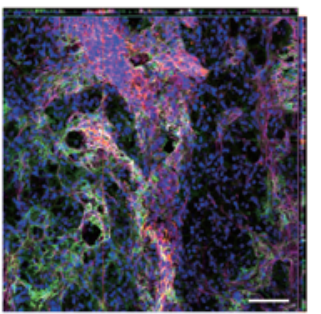

D

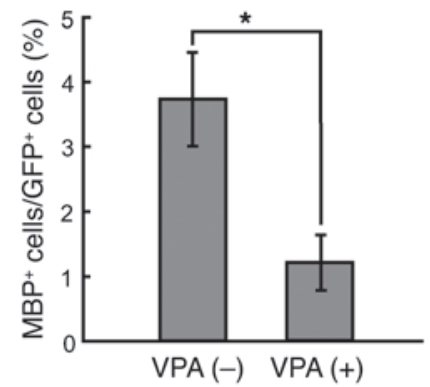

Hoechst/GFP/MAP2/GFAP

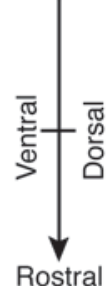

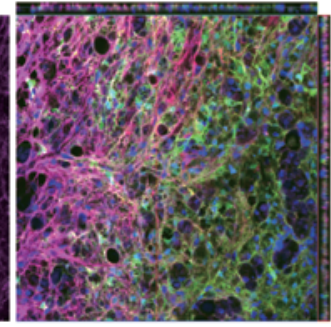

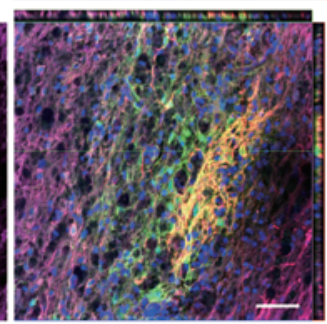

Caudal
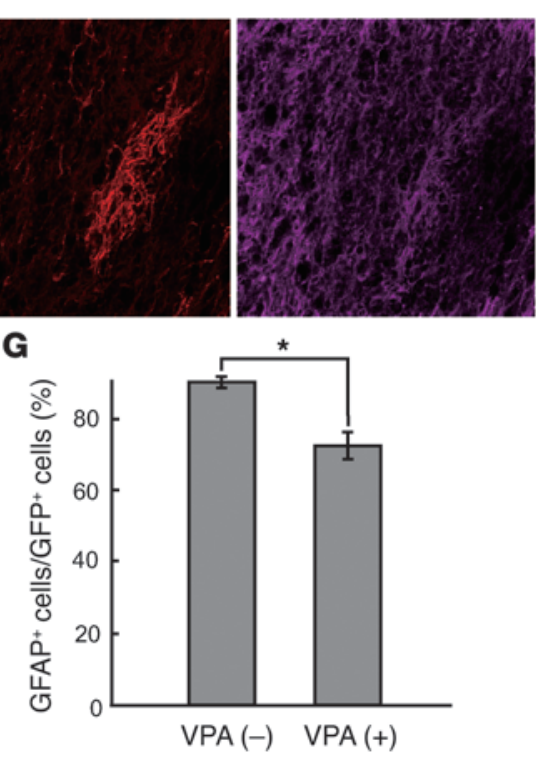


\section{Figure 3}

VPA promotes neuronal differentiation of transplanted NSCs. Representative results of GFP-NSC-transplanted SCI model mice are shown. (A) Confocal images of NSCs 1 week after transplantation into the injured spinal cords. Spinal cord sections from VPA-treated $(+)$ and untreated $(-)$ mice were stained with anti-GFP (green), anti-doublecortin (DCX) (immature neuronal marker, red) and anti-GFAP (magenta) antibodies, and Hoechst (blue). VPA administration resulted in an increase in the number of DCX-positive neuronal precursors among transplanted cells (lower panel). Scale bar: $20 \mu \mathrm{m}$. (B-D) The percentages of DCX-, GFAP-, and MBP-positive cells in GFP-positive transplanted cells were quantified. ${ }^{*} P<0.01$; ${ }^{*} P<0.05$ compared with controls (Student's $t$ test). (E) Confocal images of NSCs 5 weeks after transplantation into injured spinal cords. Spinal cord sections from VPA-treated $(+)$ and untreated (-) mice were stained with anti-GFP (green), anti-MAP2 (neuronal marker, red) and anti-GFAP (magenta) antibodies, and Hoechst (blue). VPA administration increased the numbers of MAP2-positive neurons (lower panel). Scale bar: $20 \mu \mathrm{m}$. ( $\mathbf{F}$ and $\mathbf{G}$ ) The percentages of cells positive for MAP2 or GFAP in GFP-positive transplanted cells in E were quantified. ${ }^{\star \star} P<0.01 ;{ }^{*} P<0.05$ compared with control (Student's $t$ test). All data shown in $\mathbf{B}-\mathbf{D}, \mathbf{F}$, and $\mathbf{G}$ are from at least 15 confocal images of 3 individuals in parallel experiments, with error bars representing the SD.

VPA or valpromide (VPM), an amide analog of VPA that is also an antiepileptic but is not an HDAC inhibitor (24), under differentiation culture conditions. VPA enhanced histone acetylation (Supplemental Figure 2A) and promoted neuronal differentiation and neurite outgrowth of the NSCs (Supplemental Figure 3, A-C); it also inhibited astrocytic and oligodendrocytic differentiation of NSCs (Supplemental Figure 3, D-G). A different HDAC inhibitor, trichostatin A (TSA), also enhanced histone acetylation (Supplemental Figure 2A) and neuronal differentiation of NSCs (not shown) (26). In contrast, VPM neither enhanced histone acetylation nor induced neuronal differentiation, suggesting that HDAC inhibition has an important role in regulating fate determination in NSCs.

We then assessed the histone acetylation status and differentiation profiles of transplanted NSCs. VPA administration enhanced histone acetylation in transplanted cells in the spinal cord (Supplemental Figure 2, B and C). When we examined the differentiation status of transplanted cells 1 week after transplantation, neuronal but not glial differentiation was greatly enhanced by VPA administration (Figure 3, A-D, and Supplemental Figure 4A). A similar differentiation tendency of transplanted NSCs to that at 1 week was observed at 5 weeks after transplantation: there was a dramatic increase in the number of cells positive for MAP2 (a relatively late differentiation marker of neurons in comparison with DCX) in VPA-administered mice (Figure 3, E-G, and Supplemental Figure 4B). Furthermore, VPM administration to the SCI mice neither promoted neuronal differentiation nor enhanced hind limb motor function, suggesting that HDAC inhibition has an essential role in regulating fate determination of transplanted NSCs and improvement of motor function in vivo (Supplemental Figure 5, A-C). In light of the above findings that the percentage of neurons generated from transplanted NSCs increased dramatically with VPA administration, whereas those of astrocytes and oligodendrocytes declined, we anticipated that these neurons would be likely to play a major role in regenerating the disrupted neuronal circuitry of the injured spinal cord.

Transplant-derived neurons reconstruct disrupted neuronal circuits in a relay manner. We next asked how the disrupted neuronal circuits were regenerated following the combined treatment with NSC transplantation and VPA administration. Wheat germ agglutinin (WGA), which can be transsynaptically transported, is one of the best known tracers of neural pathways (38). WGA protein can be transferred across synapses to second- and thirdorder neurons, permitting functional neuronal circuits to be tracked in the CNS. We injected WGA-expressing adenoviruses into the motor cortex of mouse brain 12 weeks after SCI. In uninjured mice, WGA was detected as intracellular granule-like structures in neurons localized in the ventral horn throughout the spinal cord (Figure 4, A and B). In untreated SCI model mice, WGA granules were almost completely absent from the caudal region below the injured site (Figure 4, A and C). Surprisingly, although we could not observe CST axonal reextension through the lesion site (Figure 1, C and D), WGA granules were clearly present in caudal large neurons located in the spinal cords of mice treated with both NSC and VPA (Figure 4, $A$ and D). Intriguingly, moreover, transplant-derived neurons in or close to the lesion site contained WGA granules (Figure $4 \mathrm{E}$ ), which were received from more rostral neurons. These data imply that WGA was conveyed through the lesion site to the caudal area via transplant-derived neurons. Considering this finding, together with the fact that WGA could be detected in caudal neurons without CST axonal reextension in mice that had undergone the combined treatment, it seemed conceivable that the transplant-derived neurons reconstructed the disrupted neuronal circuits, thereby acting as "relays" for transmitting signals between endogenous neurons whose interconnection had been abolished by the injury. In mice that received NSC transplantation alone after SCI, the percentage of WGA-positive cells among MAP2ab-positive cells in the caudal region was higher than that in untreated mice (Figure 4C) but lower than that in mice receiving combined NSC transplantation and VPA administration (Supplemental Figure 6), reflecting the degree of hind limb functional improvement (Figure 1C).

In support of the notion of a "relay" function for transplantderived neurons, immunoelectron microscopy revealed that GFPpositive transplant-derived neurons received projections from endogenous neurons (Figure 5, A and B) and that the axon terminals of transplant-derived neurons made synapses with endogenous neurons localized in the ventral horn (Figure 5, C-E).

Transplanted cells contribute directly to functional recovery of bind limb movement in SCI mice. To determine whether the transplanted cells made a direct contribution to the functional recovery of hind limbs after SCI, we performed specific ablation of transplanted cells using the toxin receptor-mediated cell knockout (TRECK) method (Figure 6A and refs. 39, 40). For this purpose, we prepared NSCs from the embryonic forebrains of GFP.LUC Tg and TR6.GFP.LUC Tg mice (Figure 6A and Supplemental Figure 7, A and B). Almost all of the transplanted TR6.GFP.LUC-NSCs were specifically ablated following DT administration (Figure 6, $B$ and $C$ ). Furthermore, after ablation of the transplanted cells, the BBB scores of SCI model mice that had undergone combined TR6.GFP.LUC-NSC transplantation and VPA administration declined rapidly to levels similar to those observed in untreated and VPA only-treated mice. These results were superimposed on the graph in Figure 1B, with the observation period extended to 12 weeks after SCI, as shown in Figure 6D (for clarity, the data for GFP-NSC.VPA and GFP.LUC-NS in Figure 1B were removed). These data indicate that the transplanted cells, in the presence of VPA, made a direct and major contribution to the functional recovery of hind limb movement in SCI model mice. 
A
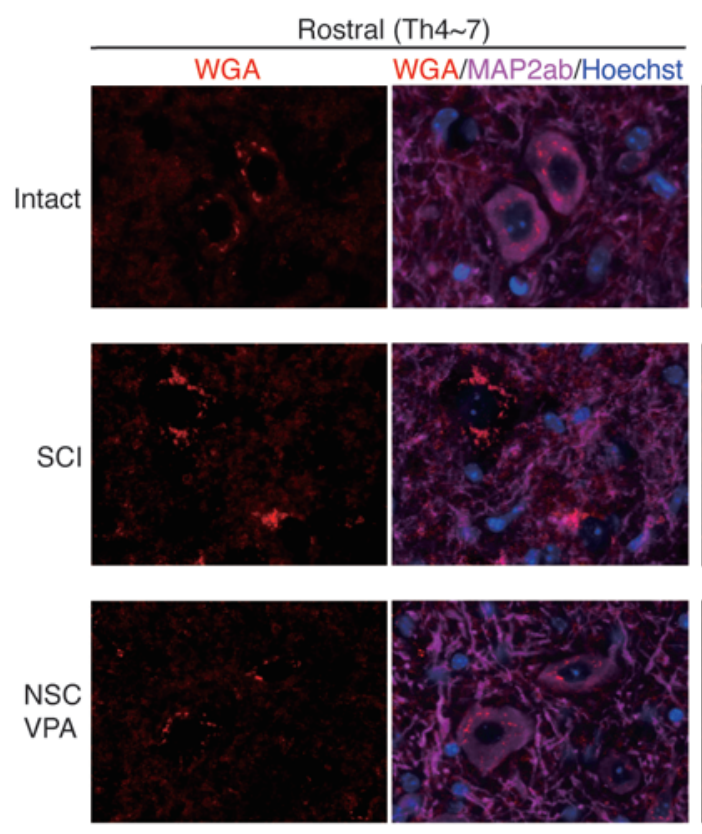

$\mathbf{E}$
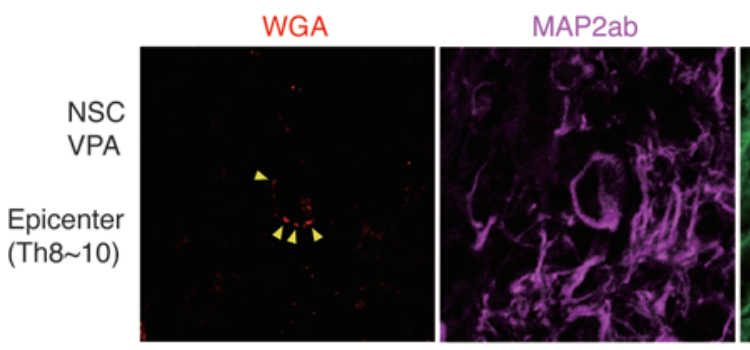
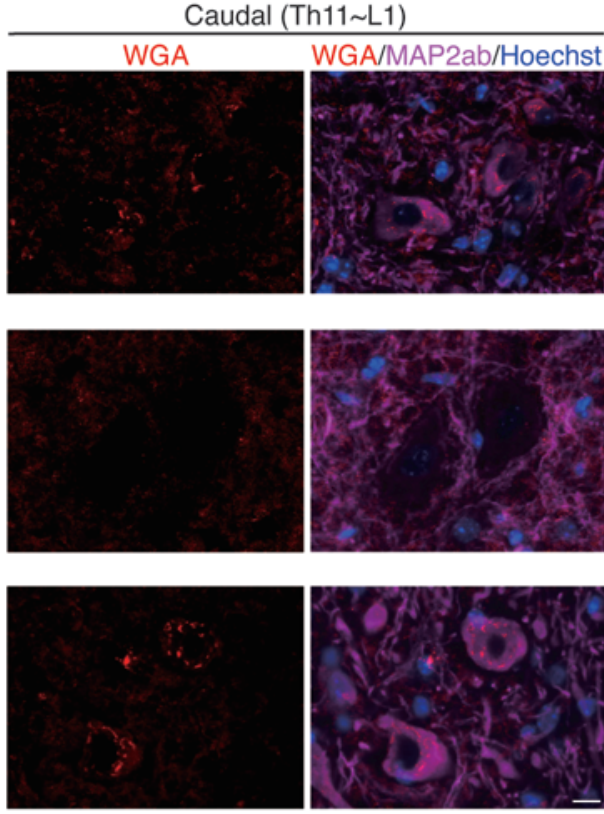
WGA/MAP2ab/Hoechst

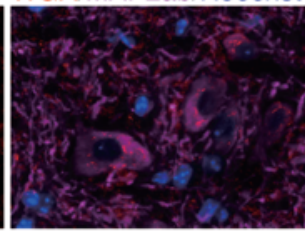

B
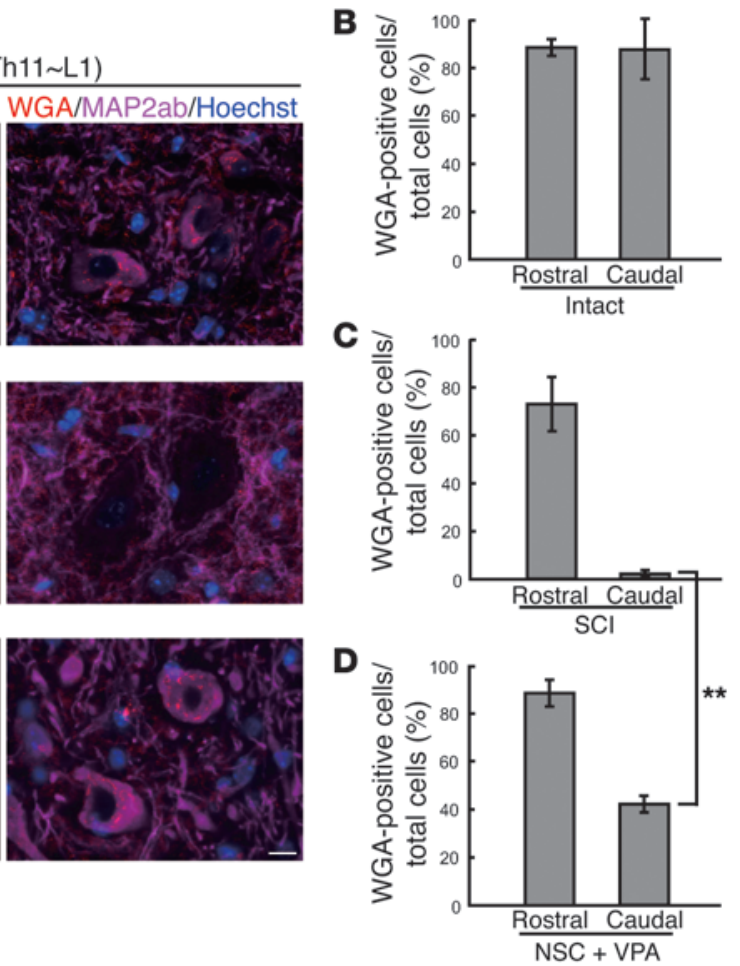

Merge/Ortho
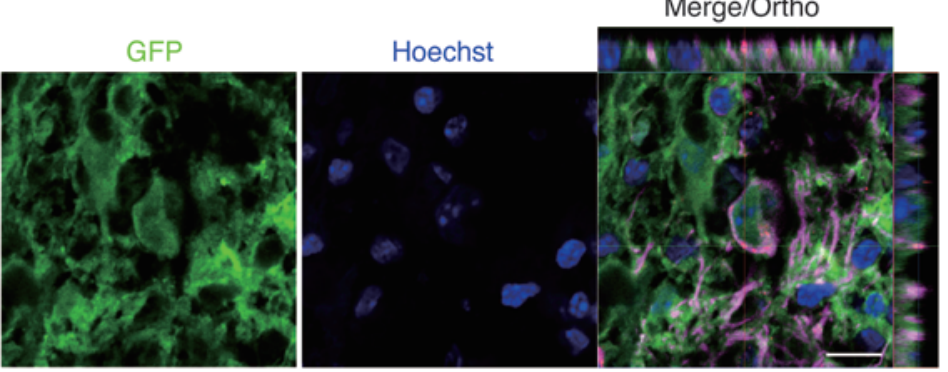

Figure 4

Transplant-derived neurons reconstruct disrupted neuronal circuits in a relay manner. (A) Representative pictures of WGA-labeled neuronal cell bodies located in the ventral horn at 14 weeks after SCI. Spinal cord sections were stained with anti-WGA (red) and -MAP2ab (magenta) antibodies and Hoechst (blue). Scale bar: $20 \mu \mathrm{m}$. Intense WGA immunoreactivity was observed as intracellular granule-like structures. Left panels show the rostral area (Th4-Th7), and right panels show the caudal area (Th11 to lumbar vertebra [L] 1). In uninjured mice, WGA injected into the bilateral motor cortices was transsynaptically transported to neurons in areas rostral and caudal to the injured site (top panels). In the SCI model mice that did not receive treatment, very little WGA was observed in caudal areas (middle panels). However, in spinal cords of animals that underwent dual treatment with NSC and VPA, WGA was clearly observed in neurons in the caudal areas (bottom panels). Representative results of GFP-NSCtransplanted SCI model mice are shown. (B-D) The percentages of WGA-positive cells in the neurons localized in the ventral horn were quantified. ${ }^{* *} P<0.05$ (Student's $t$ test). All data shown are from at least 30 images, containing more than 600 cells, from 3 individuals (5 images per area) in parallel experiments, with error bars representing SD. (E) Representative confocal images of WGA-labeled transplant-derived MAP2-positive neurons. Sections were stained with anti-WGA (red), anti-MAP2ab (magenta) and anti-GFP (green) antibodies, and Hoechst (blue). Granule-like WGA structures (yellow arrowheads) could be seen in the GFP and MAP2ab-double-positive transplant-derived neurons. Scale bar: $10 \mu \mathrm{m}$.

Both endogenous and transplant-derived local neurons play an important role in improving hind limb motor function. It has been shown recently that local neurons in the spinal cord play an important role in spontaneous functional recovery after SCI $(41,42)$. In our SCI model, we also observed slight but significant spontaneous recovery of hind limb function in untreated mice, and similar levels of recovery were sustained after ablation of transplanted cells (Figure 6D). We thus hypothesized that these recoveries were attributable to endogenous local neurons in the spinal cord. Furthermore, it seemed likely that the much higher recovery observed in mice with the combined treat- ment but without cell ablation (Figure 6D) was effected by transplant-derived local neurons in addition to the endogenous ones. To evaluate the involvement of these local neurons in our treatment regime, we divided each treated mouse group analyzed in Figure 6D into 2 subgroups (except for the "TR6.GFP.LUC-NCS-transplanted only" and "VPA-administered only" groups). The axon-sparing excitotoxin NMDA was injected at 12 weeks after SCI into the injury epicenter in the injured spinal cords of the mice in 1 subgroup for each treatment to ablate local neurons in the gray matter (43-45). In uninjured mice, NMDA injections had no significant effect on 
A

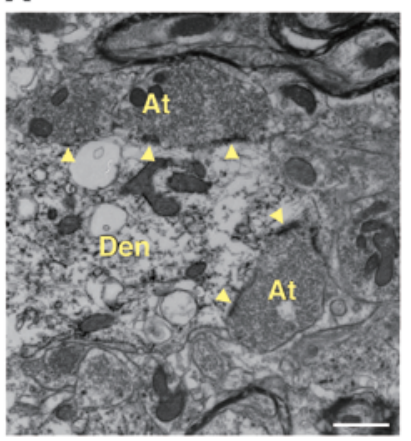

C

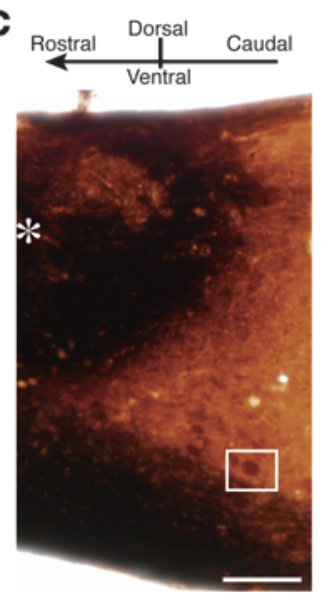

B

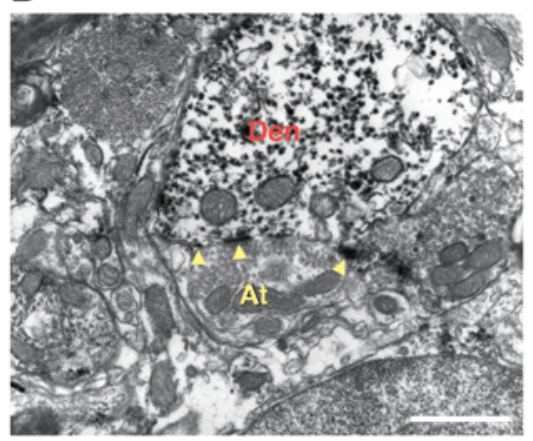

E

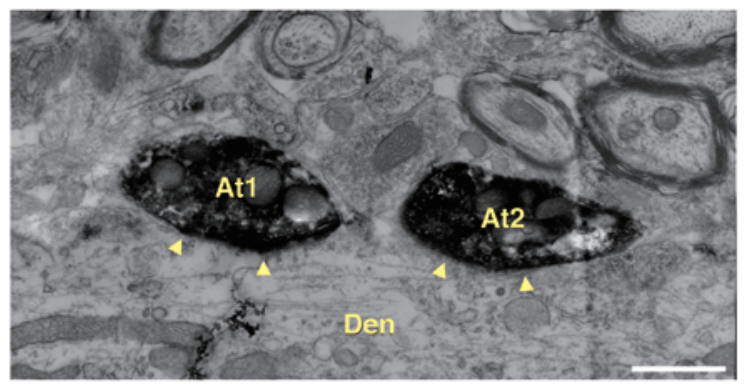

\section{Figure 5}

Transplant-derived neurons make synapses with endogenous neurons. (A) Immunoelectron microscopy image of a sagittal section of dualtreated (GFP-NSC and VPA) injured spinal cord (rostral area). A GFP-positive dendrite (Den) made synapses with GFP-negative endogenous axon termini (At) (yellow arrowheads). Scale bar: $1 \mu \mathrm{m}$. (B) In other rostral regions, a dendrite of a GFP-positive transplant-derived neuron made a synapse (yellow arrowheads) with the axon terminus of a GFP-negative endogenous neuron. Scale bar: 1 um. (C) Sagittal section of dualtreated (NSC and VPA) injured spinal cord (caudal area) stained with anti-GFP antibody (dark brown). The epicenter of the SCl is indicated (*). Scale bar: $500 \mu \mathrm{m}$. (D) High-magnification image of a large neuron localized in the ventral horn in the white rectangle in C. GFP-positive transplanted neurons extended their processes toward an endogenous neuron (yellow arrowheads). Scale bar: $100 \mu \mathrm{m}$. (E) Immunoelectron microscopy image of the boxed area in D. GFP-positive axon termini made synapses with the dendrite of a GFP-negative endogenous large neuron (yellow arrowheads). Scale bar: $1 \mu \mathrm{m}$.

hind limb function (data not shown). However, as shown in Figure 6E, NMDA injections completely reversed both spontaneous and treatment-provoked functional recovery of hind limb movement in SCI model mice, indicating that both endogenous and transplantderived local neurons indeed play an important role in the restoration of hind limb motor function.

\section{Discussion}

Over the last 3 decades, numerous strategies to cure CNS injuries, including SCI, have been devised and tested (1-18). Regeneration of injured neuronal circuits in the adult CNS is a multistep process: axons must extend their processes to contact appropriate neuronal targets and must then be remyelinated, and functional synapses need to form on the surfaces of the targeted neurons. Stem cellderived cell supplementation to the injured spinal cord was previously shown to induce partial recovery $(8,9,11,15)$. However, the SCI models used in these studies involved moderate injury, so that hind limb motor function recovered spontaneously and sufficiently, even in untreated subjects, for the animals to be able to support their own weight and walk (BBB scores were approximately 10 to 13 ) within 3 weeks. In these studies, axons demyelinated by the injury were reportedly remyelinated by transplant-derived oligodendrocytes, leading to significant but minor improvements in BBB score (to approximately 12 to 16 ) over those observed in untreated mice.

In clinical practice, as treatment of moderate SCI has improved greatly with advanced surgical reconstruction techniques and better general management, the development of regenerative therapies for severer, recalcitrant forms of SCI is more pressing (46). Accordingly, we applied in this study a severe SCI model in which CST axons were completely disrupted (Figure 1C) to assess the effectiveness of our strategy. Hind limb motor function in untreated mice recovered only marginally (to a BBB score of $\sim 4$ ), whereas recovery improved dramatically, to the level at which mice could support their weight, as a consequence of the combined treatment with NSCs and VPA (BBB score $\sim 9$ to 10). Such a degree of improvement in hind limb function in severely injured mice has, to the best of our knowledge, never before been attained. Although a neuroprotective effect has been reported for VPA (27-31), this does not seem to be pertinent to the functional recovery of SCI because mice treated with VPA alone showed no 

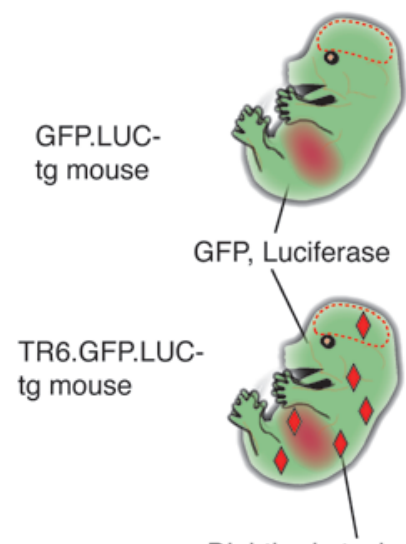

Diphtheria toxin receptor

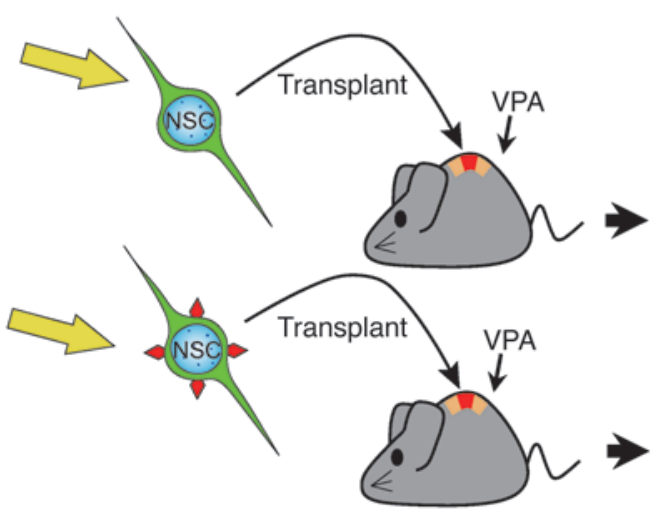

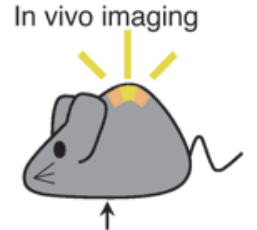

Luciferin i.p.

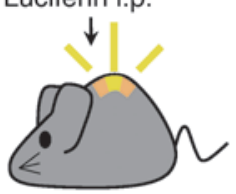

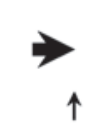

Diphtheria toxin i.p.

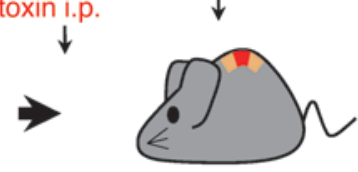

BBB score check

In vivo imaging

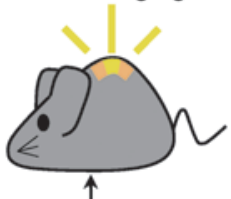

Luciferin i.p.

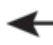

B

GFP.

LUC

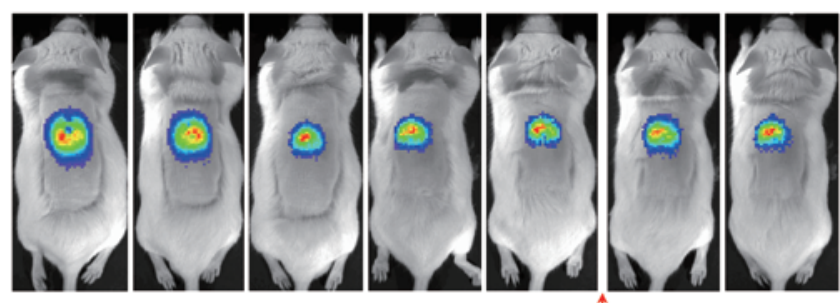

Diphtheria toxin i.p. (50 mg/kg $\times 2$ days)

TR6.

GFP.

LUC

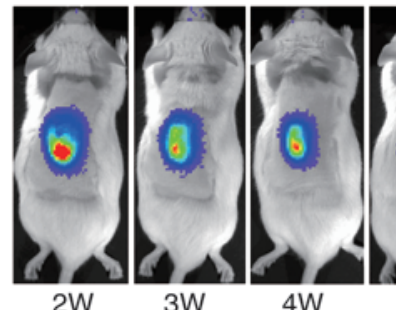

$3 \mathrm{~W}$

$4 \mathrm{~W}$

D

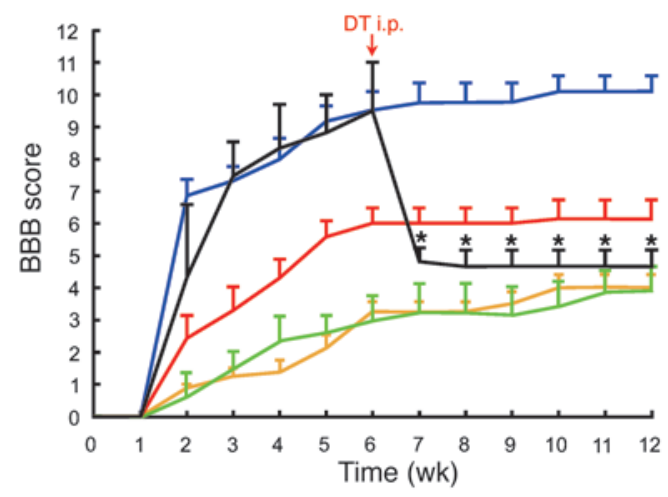

— GFP.LUC-NSC.VPA.DT, $n=9$

- TR6.GFP.LUC-NSC.VPA.DT, $n=6$

- TR6.GFP.LUC-NSC, $n=6$

- VPA, $n=8$

- No treatment, $n=8$

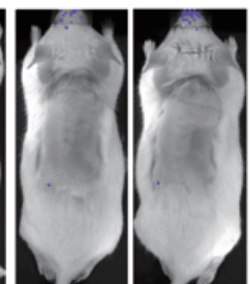

$8 \mathrm{~W}$
C
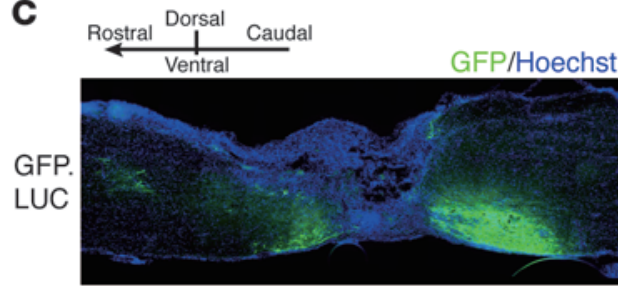

TR6.

GFP.

LUC
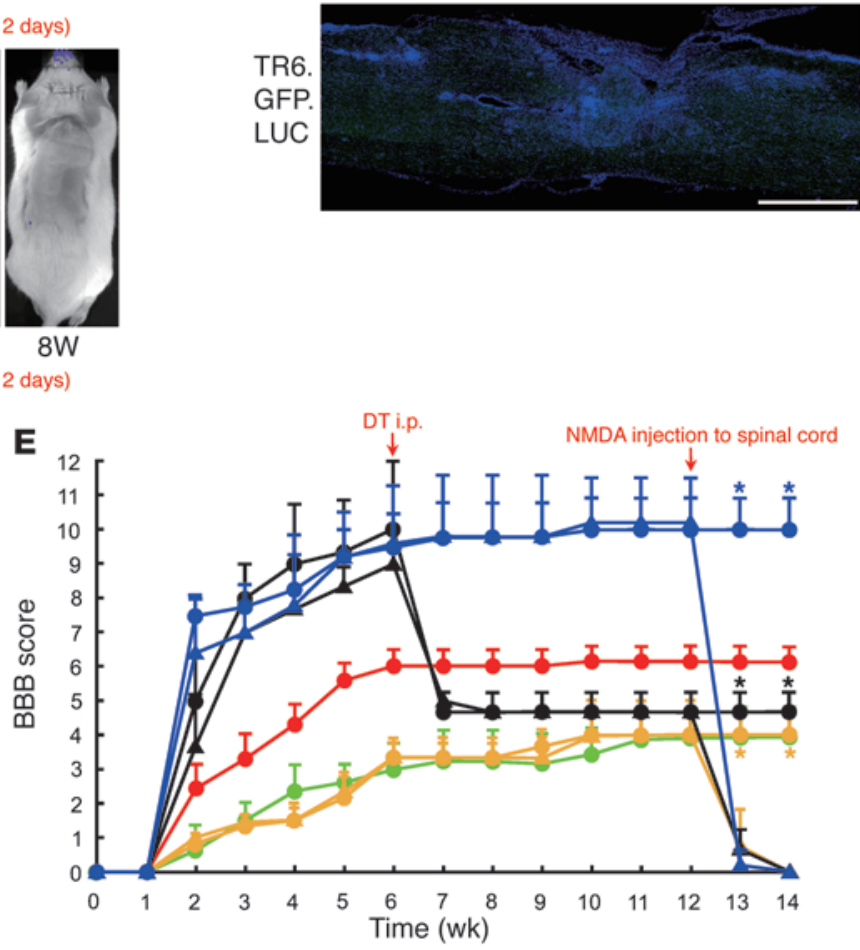

$\rightarrow$ GFP.LUC-NSC.VPA.DT, $n=4$

- GFP.LUC-NSC.VPA.DT.NMDA, $n=5$

$\rightarrow$ TR6.GFP.LUC-NSC.VPA.DT, $n=3$

— TR6.GFP.LUC-NSC.VPA.DT.NMDA, $n=3$

$\rightarrow$ TR6.GFP.LUC-NSC, $n=6$

- VPA, $n=8$

$\rightarrow$ No treatment, $n=5$

No treatment.NMDA, $n=3$ 


\section{Figure 6}

Ablation of transplanted cells abolishes hind limb motor function recovery. (A) Schematic of the protocols for NSC transplantation and for detection and ablation of transplanted cells. NSCs derived from GFP. LUC- or TR6.GFP.LUC-Tg mice were transplanted into SCI model mice 1 week after injury. VPA was intraperitoneally administered every day for 1 week. Survival of transplanted cells and locomotor function of the mice were monitored weekly for 14 weeks. (B) Survival of transplanted cells was checked every week using a bioluminescence imaging system. 6 weeks after injury ( 5 weeks after transplantation), each mouse received 2 DT administrations. By the following week, LUC activity had completely disappeared in mice transplanted with TR6.GFP.LUCNSCs (lower panel). (C) Sagittal sections from SCI model mice transplanted with GFP.LUC- and TR6.GFP.LUC-NSCs 2 weeks after DT injection. All transplanted cells were ablated with DT (lower panel). Scale bar: $1 \mathrm{~mm}$. (D) Time course of the changes in BBB scores in SCI model mice. The hind limb function of mice that had undergone dual treatment with TR6.GFP.LUC-NSCs and VPA dropped drastically after DT administration (black line). ${ }^{*} P<0.0001$ compared with GFP.LUCNSC-transplanted, VPA-administered, and DT-injected SCI model mice (blue line) (repeated measures ANOVA). Data are mean \pm SEM. VPA, $n=8$; no treatment, $n=8$. (E) Twelve weeks after injury, groups of $\mathrm{SCl}$ model mice received NMDA injections, as indicated, into the injury epicenter, to ablate local neurons in the gray matter (blue, black, and yellow lines with triangles). ${ }^{*} P<0.0001$ compared with nonNMDA-injected mice in each group (blue, black, and yellow lines with circles) (repeated measures ANOVA). Data represent mean \pm SEM.

greater improvement compared with untreated mice (Figures 1 and 6). We suggest that, in severe SCI, supplementation with neurons may be more beneficial than supplementation with oligodendrocytes for reconstructing destroyed neuronal circuits. Our observation that transplant-derived neuronal axons could be remyelinated by endogenous oligodendrocytes (Supplemental Figure 9) suggests that the injured CNS may have a much greater restorative capacity than previously thought.

Differentiation of NSCs transplanted into the injured spinal cord is generally restricted to the glial lineage: the host spinal cord milieu is not permissive for neuronal differentiation $(7,21$, $22,47,48)$. Neuronal differentiation of neuronal lineage-committed precursors grafted into the injured spinal cord is also severely inhibited, whereas the same cells can differentiate efficiently into neurons under in vitro culture conditions as well as in the intact spinal cord $(47,48)$. In an earlier attempt to stimulate neuronal differentiation, NSCs transduced with the proneural transcription factor neurogenin 2 (Neurog2) were engrafted into injured spinal cords, resulting in moderate functional recovery of hind limbs in comparison with untreated animals (11). The authors concluded, however, that oligodendrocytes derived unexpectedly from the transduced NSCs contributed to the improvement of motor function by remyelinating the injured axons. Although Neurog2-transduced NSCs actually differentiated more efficiently into neurons than into oligodendrocytes after transplantation, the roles of these neurons were not thoroughly investigated.

In the present study, we adopted what we believe is a novel approach for the treatment of SCI: manipulation of transplanted NSC fate by an epigenetic reagent. A combined treatment involving NSC transplantation and administration of the HDAC inhibitor VPA led to a marked functional recovery. We will refer below to this dual treatment as the HINT ( transplantation) method. Immunohistochemical analysis revealed that VPA administration promoted the neuronal differentiation of transplanted NSCs. We examined extensively the roles of the neurons responsible for reconstruction of broken neuronal networks using 2 neuronal tracers, immunoelectron microscopy, and 2 cellablation methods. These results revealed that transplant-derived neurons received projections from endogenous neurons and that their extended processes made synapses with endogenous neurons in the ventral horn. Furthermore, we showed clearly that transplant-derived neurons reconstructed the disrupted CST, allowing the restoration of signal transmission in a relay manner (Figure 7). Among these transplant-derived neurons, $16.9 \% \pm 2.3 \%$ were vesicular glutamate transporter (VGLUT) 2-positive (glutamatergic) and $70.1 \% \pm 1.8 \%$ were glutamic acid decarboxylase (GAD) 65 positive (GABAergic) (Supplemental Figure 10). Although we have not examined the neuronal subtypes of the remaining $13 \%$, these data suggest that both excitatory and inhibitory neuronal subtypes were generated from transplanted NSCs. In light of these findings, we hypothesize that the balance between the transplant-derived excitatory and inhibitory neuronal subtypes is important for hind limb functional recovery after SCI. However, we must await further investigation to substantiate this hypothesis.

Although recovery was not as great as it was in mice that underwent the HINT method, mice treated with NSCs alone showed greater improvement of hind limb function compared with the untreated controls, which exhibited slight but significant restoration (Figures 1 and 6). This is probably due to trophic support provided by transplanted cells to endogenous cells, as suggested in previous reports (12-14), since transplanted NSCs barely differentiate into neurons in the injured spinal cord. However, any beneficial trophic effect of transplanted NSCs on endogenous neurons is unlikely to be sufficient to explain the functional recovery of the injured spinal cord because we did not observe axon reextension beyond the lesion site in our severe SCI model even in mice treated by the HINT method. Thus, it is likely that local neurons in the spinal cord (perhaps a mixture of preserved endogenous neurons and transplant-derived neurons) transmit signals in a relay manner. This is also supported by the fact that both spontaneous and treatment-provoked hind limb functional restoration were completely reversed by NMDA injection to ablate local neurons in the spinal cord (Figure 6E).

As an established drug for long-term therapy for epilepsy, VPA may be more appropriate for use in clinical trials than other drugs or ectopically expressed Tgs. Nonetheless, while the combination of epigenetic manipulation and NSC transplantation for the treatment of SCI yielded a dramatic functional improvement in hind limb movement, recovery was incomplete. To achieve a more efficient treatment for SCI, administration of a more complex "cocktail" (combinatorial treatment in conjunction with other strategies, such as neurotrophin-mediated prevention of neuronal death, regeneration of axons by suppressing myelin inhibitors, and remyelination) may be necessary.

\section{Methods}

$\mathrm{Tg}$ mice. For visualization and specific ablation of transplanted cells, we used the TRECK method (40). To achieve more specific and conditional ablation of target cells, we introduced 2 point mutations (I117V/L148V) in human HB-EGF (39). The modified human HB-EGF (I117V/L148V) gene fragment fused with $E G F P$ was subcloned into EcoRI sites in the mammalian expression vector pCAGGS (49) (pCAG-TR6/GFP). The 3.8-kb SpeIHindIII fragment was isolated from pCAG-TR6/GFP and microinjected into fertilized mouse eggs (C57BL/6J Jcl background). These eggs were 
A

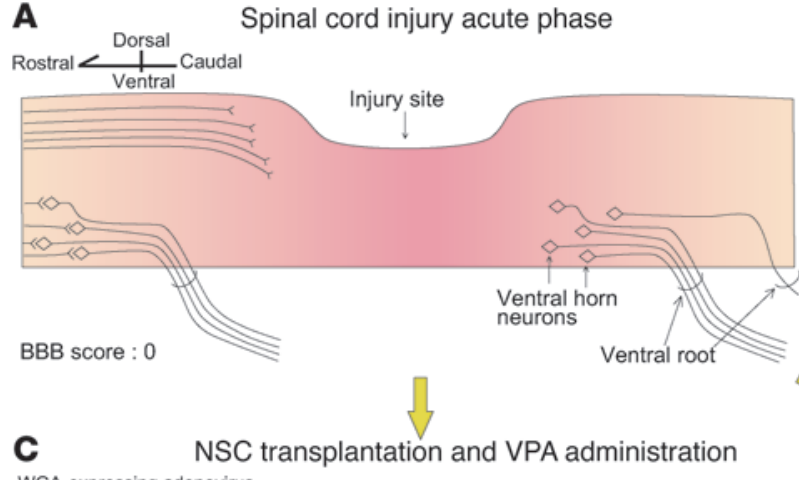

WGA-expressing adenovirus

injection into motor cortex

灙湿

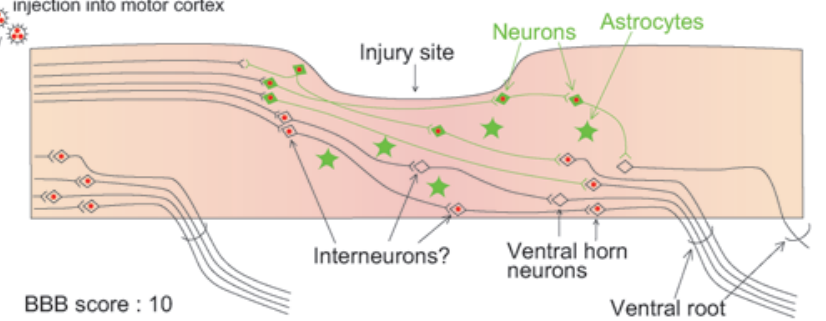

B

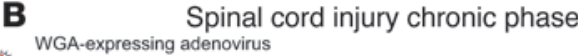

WGA-expressing adenovirus
漈瀿 injection into motor cortex

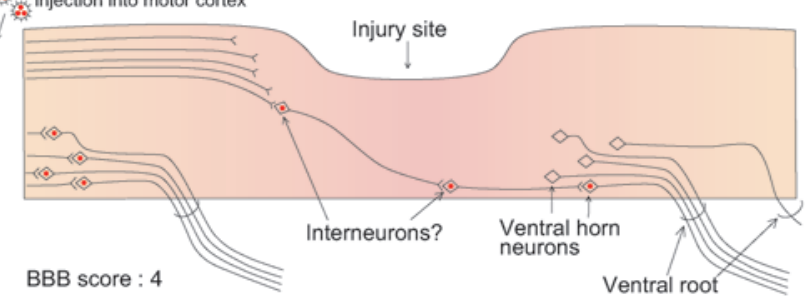

BBB score : 4

D

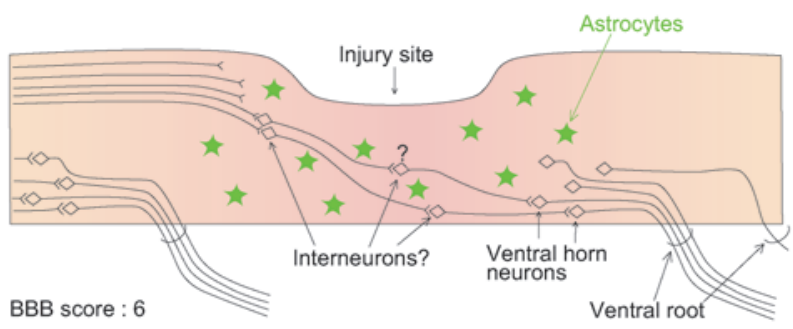

$\mathbf{F}$

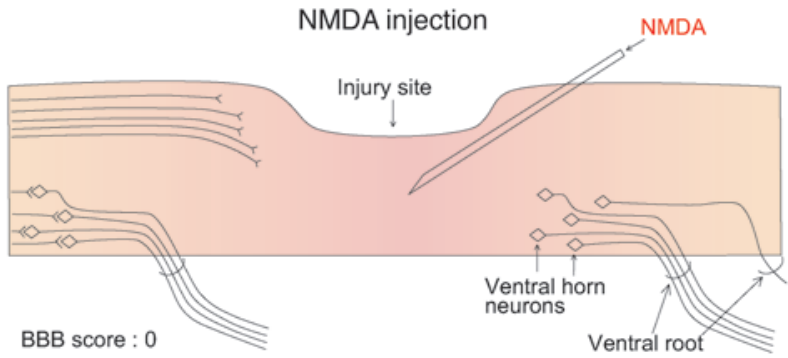

\section{Figure 7}

Diagrammatic summary of destruction and reconstruction of neural circuits in the injured spinal cord. (A) In the acute phase of SCI, CST fibers are disrupted and hind limb movement is completely lost. (B) In the chronic phase, WGA transport through the lesion site to the caudal area is negligible, probably because very few CST fibers are restored. (D) When NSCs were transplanted to the injured spinal cord, almost all of them differentiated into astrocytes, with the result that very little restoration of the injured CST occurred. (C) Following VPA administration, neuronal differentiation of transplanted NSCs was greatly enhanced and dramatic functional recovery could be observed. WGA is conveyed through the lesion site to the caudal area via transplant-derived neurons. (E) Ablation of transplant-derived cells with DT nullified hind limb functional recovery, suggesting that the transplanted cells contribute directly to the functional recovery in hind limbs. (F) Ablation of residual local neurons in the lesion site by NMDA led to a complete impairment of hind limb movement, suggesting that residual local endogenous neurons also play an important role in the improvement of hind limb motor function.

subsequently implanted into pseudopregnant female mice (C57BL/6J Jcl) to obtain the Tg mouse line TR6.GFP-Tg. Female mice homozygous for the CAG-LUC Tg (LUC-Tg) were mated with TR6.GFP-Tg heterozygous or CAG-GFP (GFP-Tg) (33) heterozygous male mice. E14.5 forebrainderived NSCs from mice heterozygous for GFP (GFP-NSCs), TR6.GFP, and LUC (TR6.GFP.LUC-NSCs) or for GFP and LUC (GFP.LUC-NSCs) were expanded and used for transplantation. Tg mice expressing GFP under the control of the CAG promoter were a gift from M. Okabe (Osaka University, Suita, Osaka, Japan).

Cell culture. E14.5 mouse forebrains were dissected and triturated in calcium- and magnesium-free HBSS and cultured on poly-ornithine/ fibronectin-coated (O/F-coated) dishes for 4 days in N2-supplemented DMEM/F-12 containing $10 \mathrm{ng} / \mathrm{ml}$ bFGF (PeproTech). Cells were replated on $\mathrm{O} / \mathrm{F}$-coated dishes in NS-A medium (Euroclone) supplemented with modified $\mathrm{N} 2$ and $10 \mathrm{ng} / \mathrm{ml}$ each of EGF (PeproTech) and bFGF
(NS expansion medium) (36). We routinely used HBSS to detach cells from the dish; we split cells 1:3 and replated them onto O/F-coated dishes every 2 days. Cells that had been passaged 5-10 times were used for transplantation. See Supplemental Methods for details of NSC differentiation in vitro.

SCI model. All animal studies were conducted with the approval of the Nara Institute of Science and Technology animal care and use committee. We used 15-week-old male ICR mice weighing 37-45 g. Anesthetized (sodium pentobarbital, $40 \mathrm{mg} / \mathrm{kg}$ body weight) mice received laminectomies and partial laminectomies at the ninth and tenth thoracic spinal vertebrae, respectively. The dorsal surface of the dura mater was exposed and SCI was applied using an SCI device (90 kdyn; Infinite Horizon impactor; Precision Systems \& Instrumentation) as previously described (8). The muscle and skin were closed in layers. The mice underwent manual bladder evacuation once a day. We checked the lesion site sizes in several SCI mice 1 week 
after injury and confirmed that they were comparable. One week after the injury, hind limb movement in the model mice was not observed (BBB score: 0 ). Mice with incomplete injury (BBB score above 0 at 1 week after injury) were excluded (2.4\%).

Transplantation. Seven days after injury, mice were anesthetized and transplanted with NSCs using a glass micropipette attached to a stereotaxic injector (Narishige). The tip of the micropipette was inserted into the injury epicenter in the injured spinal cord, and $2 \mu \mathrm{l}$ of NS medium lacking growth factor, with or without NSCs $\left(0.5 \times 10^{6} / \mu \mathrm{l}\right)$, was injected at a rate of $1 \mu \mathrm{l} / \mathrm{min}$. All mice subcutaneously received cyclosporine immunosuppressant $(10 \mathrm{mg} / \mathrm{kg})$ and gentamicin $(8 \mathrm{mg} / \mathrm{kg})$ daily. After transplantation, mice were given daily intraperitoneal injections of VPA $(150 \mathrm{mg} / \mathrm{kg})$ or saline (control) for the following 7 days.

Behavioral testing. We evaluated the motor function of the hind limbs for up to 14 weeks after injury. See Supplemental Methods for details.

Immunocytochemistry. Immunocytochemical experiments were performed as described previously (7). See Supplemental Methods for antibodies used and further details.

Immunohistochemistry. We performed immunohistochemical analysis on tissue sections using 3 or more independent samples from the mice. See Supplemental Methods for details.

Immunoelectron microscopy. See Supplemental Methods.

Anterograde labeling of the CST. Twelve weeks after injury, descending CST fibers were labeled with BDA ( $10 \%$ in saline, $2 \mu$ l per cortex, MW 10,000; Molecular Probes) $(6,16,17)$ by injection into the left and right motor cortices (50) (for 3 uninjured mice, 3 mice that received SCI with no treatment, and 3 mice that received SCI and underwent combined treatment with NSCs and VPA). See Supplemental Methods for details.

Visualization of multisynaptic neuronal pathways. To visualize selective and functional transsynaptic neuronal pathways, WGA-expressing recombinant adenoviruses (provided by Y. Yoshihara, RIKEN Brain Science Institute, Wako, Saitama, Japan) were used (38). In this system, injections of WGAexpressing adenovirus in well-mapped neural pathways result in labeling of first-, second-, and third-order neurons. WGA protein is efficiently transported in axons and dendrites in both anterograde and retrograde directions. See Supplemental Methods for details.

In vivo imaging of transplanted cells. In vivo imaging experiments were performed as described previously (8). See Supplemental Methods for details.
Ablation of transplant-derived cells. Cell ablation experiments were performed as described previously $(39,40)$. See Supplemental Methods for details.

Ablation of local neurons. To ablate neurons localized in the gray matter, NMDA was injected into the spinal cord at thoracic vertebrae 9 (Th9). Twelve weeks after injury, SCI model mice (5 GFP.LUC-NSC-transplanted and VPA-treated mice, 3 TR6.GFP.LUC-NSC-transplanted and VPA-treated mice, and 3 untreated mice) received bilateral injections of NMDA into 2 sites of the spinal cord $(0.5 \mathrm{ml}$ of $10 \mathrm{mM}$ in saline per site) under anesthesia.

Statistics. We performed statistical analysis with an unpaired 2-tailed Student's $t$ test for single comparisons. For analysis of the open-field scores, we used repeated measures ANOVA (Prism, GraphPad). $P<0.05$ was considered significant.

\section{Acknowledgments}

We thank Y. Yoshihara for providing the WGA-expressing adenovirus and $\mathrm{M}$. Okabe for donating the Tg mice expressing GFP under the control of the CAG promoter. We also thank Y. Bessho, T. Matsui, S. Komai, M. Arai, H. Fujioka, T. Yamashita, M. Ueno, Y. Sasai, K. Muguruma, B. Juliandi, K. Yone, T. Setoguchi, and Y. Fujimoto for valuable discussions and technical advice; I. Smith for editing the manuscript; and N. Ueda and M. Tano for secretarial assistance. This work was supported by the Naito Foundation, the NAIST Cross-Fields Collaboration Promoting Project, and by a grant-in-aid for Exploratory Research, a grant-inaid for Young Scientists (B), and the NAIST Global COE Program (Frontier Biosciences: Strategies for Survival and Adaptation in a Changing Global Environment) from the Ministry of Education, Culture, Sports, Science, and Technology of Japan.

Received for publication March 11, 2010, and accepted in revised form July 7, 2010.

Address correspondence to: Kinichi Nakashima, Laboratory of Molecular Neuroscience, Graduate School of Biological Sciences, Nara Institute of Science and Technology, 8916-5 Takayama, Ikoma 631-0192, Japan. Phone: 81.743.72.5471; Fax: 81.743.72.5479; E-mail: kin@bs.naist.jp.
1. Thuret S, Moon LD, Gage FH. Therapeutic interventions after spinal cord injury. Nat Rev Neurosci. 2006;7(8):628-643

2. David S, Aguayo AJ. Axonal elongation into peripheral nervous system "bridges" after central nervous system injury in adult rats. Science. 1981;214(4523):931-933.

3. Richardson PM, McGuinness UM, Aguayo AJ. Axons from CNS neurons regenerate into PNS grafts. Nature. 1980;284(5753):264-265.

4. Schnell L, Schwab ME. Axonal regeneration in the rat spinal cord produced by an antibody against myelin-associated neurite growth inhibitors. Nature. 1990;343(6255):269-272.

5. Bregman BS, Kunkel-Bagden E, Schnell L, Dai HN, Gao D, Schwab ME. Recovery from spinal cord injury mediated by antibodies to neurite growth inhibitors. Nature. 1995;378(6556):498-501.

6. Bradbury EJ, et al. Chondroitinase $\mathrm{ABC}$ promotes functional recovery after spinal cord injury. Nature. 2002;416(6881):636-640.

7. Setoguchi T, et al. Treatment of spinal cord injury by transplantation of fetal neural precursor cells engineered to express BMP inhibitor. Exp Neurol. 2004;189(1):33-44.

8. Okada S, et al. In vivo imaging of engrafted neural stem cells: its application in evaluating the optimal timing of transplantation for spinal cord injury. Faseb J. 2005;19(13):1839-1841.

9. Cummings BJ, et al. Human neural stem cells differentiate and promote locomotor recovery in spinal cord-injured mice. Proc Natl Acad Sci U S A. 2005;102(39):14069-14074.

10. Ogawa Y, et al. Transplantation of in vitroexpanded fetal neural progenitor cells results in neurogenesis and functional recovery after spinal cord contusion injury in adult rats. J Neurosci Res. 2002;69(6):925-933.

11. Hofstetter CP, et al. Allodynia limits the usefulness of intraspinal neural stem cell grafts; directed differentiation improves outcome. Nat Neurosci. 2005;8(3):346-353.

12. Shumsky JS, Tobias CA, Tumolo M, Long WD, Giszter SF, Murray M. Delayed transplantation of fibroblasts genetically modified to secrete BDNF and NT-3 into a spinal cord injury site is associated with limited recovery of function. Exp Neurol. 2003;184(1):114-130.

13. Tobias CA, et al. Delayed grafting of BDNF and NT-3 producing fibroblasts into the injured spinal cord stimulates sprouting, partially rescues axotomized red nucleus neurons from loss and atrophy, and provides limited regeneration. Exp Neurol. 2003;184(1):97-113.
14. Yan J, Welsh AM, Bora SH, Snyder EY, Koliatsos VE. Differentiation and tropic/trophic effects of exogenous neural precursors in the adult spinal cord. J Comp Neurol. 2004;480(1):101-114.

15. Keirstead HS, et al. Human embryonic stem cellderived oligodendrocyte progenitor cell transplants remyelinate and restore locomotion after spinal cord injury. J Neurosci. 2005;25(19):4694-4705.

16. Hata K, et al. RGMa inhibition promotes axonal growth and recovery after spinal cord injury. $J$ Cell Biol. 2006;173(1):47-58.

17. Kaneko S, et al. A selective Sema3A inhibitor enhances regenerative responses and functional recovery of the injured spinal cord. Nat Med. 2006;12(12):1380-1389.

18. Mi S, et al. LINGO-1 antagonist promotes spinal cord remyelination and axonal integrity in MOGinduced experimental autoimmune encephalomyelitis. Nat Med. 2007;13(10):1228-1233.

19. Nakamura M, Houghtling RA, MacArthur L, Bayer BM, Bregman BS. Differences in cytokine gene expression profile between acute and secondary injury in adult rat spinal cord. Exp Neurol. 2003;184(1):313-325.

20. Setoguchi T, et al. Traumatic injury-induced BMP7 expression in the adult rat spinal cord. Brain Res. 2001;921(1-2):219-225. 
21. Martino G, Pluchino S. The therapeutic potential of neural stem cells. Nat Rev Neurosci. 2006; $7(5): 395-406$.

22. Abematsu M, Smith I, Nakashima K. Mechanisms of neural stem cell fate determination: extracellular cues and intracellular programs. Curr Stem Cell Res Ther. 2006;1(2):267-277.

23. Blaheta RA, Cinatl J Jr. Anti-tumor mechanisms of valproate: a novel role for an old drug. Med Res Rev. 2002;22(5):492-511.

24. Gottlicher $M$, et al. Valproic acid defines a novel class of HDAC inhibitors inducing differentiation of transformed cells. Embo J. 2001;20(24):6969-6978.

25. Phiel CJ, Zhang F, Huang EY, Guenther MG, Lazar MA, Klein PS. Histone deacetylase is a direct target of valproic acid, a potent anticonvulsant, mood stabilizer, and teratogen. J Biol Chem. 2001;276(39):36734-36741.

26. Hsieh J, Nakashima K, Kuwabara T, Mejia E, Gage FH. Histone deacetylase inhibition-mediated neuronal differentiation of multipotent adult neural progenitor cells. Proc Natl Acad Sci U S A. 2004;101(47):16659-16664.

27. Hashimoto R, Hough C, Nakazawa T, Yamamoto $\mathrm{T}$, Chuang DM. Lithium protection against glutamate excitotoxicity in rat cerebral cortical neurons: involvement of NMDA receptor inhibition possibly by decreasing NR2B tyrosine phosphorylation. J Neurochem. 2002;80(4):589-597.

28. Kanai H, Sawa A, Chen RW, Leeds P, Chuang DM. Valproic acid inhibits histone deacetylase activity and suppresses excitotoxicity-induced GAPDH nuclear accumulation and apoptotic death in neurons. Pharmacogenomics J. 2004;4(5):336-344.

29. Ren M, Leng Y, Jeong M, Leeds PR, Chuang DM. Valproic acid reduces brain damage induced by transient focal cerebral ischemia in rats: potential roles of histone deacetylase inhibition and heat shock protein induction. J Neurochem.
2004;89(6):1358-1367.

30. Kim AJ, Shi Y, Austin RC, Werstuck GH. Valproate protects cells from ER stress-induced lipid accumulation and apoptosis by inhibiting glycogen synthase kinase-3. J Cell Sci. 2005;118(pt 1):89-99.

31. Peng GS, et al. Valproate pretreatment protects dopaminergic neurons from LPS-induced neurotoxicity in rat primary midbrain cultures: role of microglia. Brain Res Mol Brain Res. 2005;134(1):162-169.

32. Yuan PX, Huang LD, Jiang YM, Gutkind JS, Manji HK, Chen G. The mood stabilizer valproic acid activates mitogen-activated protein kinases and promotes neurite growth. J Biol Chem. 2001;276(34):31674-31683.

33. Okabe M, Ikawa M, Kominami K, Nakanishi T, Nishimune Y. 'Green mice' as a source of ubiquitous green cells. FEBS Lett. 1997;407(3):313-319.

34. Johe KK, Hazel TG, Muller T, Dugich-Djordjevic MM, McKay RD. Single factors direct the differentiation of stem cells from the fetal and adult centra nervous system. Genes Dev. 1996;10(24):3129-3140.

35. Nakashima K, et al. Synergistic signaling in fetal brain by STAT3-Smad1 complex bridged by p300. Science. 1999;284(5413):479-482.

36. Conti L, et al. Niche-independent symmetrical selfrenewal of a mammalian tissue stem cell. PLoS Biol. 2005;3(9):e283.

37. Basso DM, Beattie MS, Bresnahan JC. A sensitive and reliable locomotor rating scale for open field testing in rats. J Neurotrauma. 1995;12(1):1-21.

38. Kinoshita N, Mizuno T, Yoshihara Y. Adenovirusmediated WGA gene delivery for transsynaptic labeling of mouse olfactory pathways. Chem Senses. 2002;27(3):215-223.

39. Furukawa N, Saito M, Hakoshima T, Kohno K. A diphtheria toxin receptor deficient in epiderma growth factor-like biological activity. J Biochem. 2006;140(6):831-841.

40. Saito M, et al. Diphtheria toxin receptor-mediated conditional and targeted cell ablation in transgenic mice. Nat Biotechnol. 2001;19(8):746-750.

41. Bareyre FM, Kerschensteiner M, Raineteau O, Mettenleiter TC, Weinmann O, Schwab ME. The injured spinal cord spontaneously forms a new intraspinal circuit in adult rats. Nat Neurosci. 2004;7(3):269-277.

42. Courtine G, et al. Recovery of supraspinal control of stepping via indirect propriospinal relay connections after spinal cord injury. Nat Med. 2008;14(1):69-74.

43. Faulkner JR, Herrmann JE, Woo MJ, Tansey KE, Doan NB, Sofroniew MV. Reactive astrocytes protect tissue and preserve function after spinal cord injury. J Neurosci. 2004;24(9):2143-2155.

44. Agrawal SK, Fehlings MG. Role of NMDA and non-NMDA ionotropic glutamate receptors in traumatic spinal cord axonal injury. J Neurosci. 1997;17(3):1055-1063.

45. Yu SP, Yeh C, Strasser U, Tian M, Choi DW. NMDA receptor-mediated $\mathrm{K}+$ efflux and neuronal apoptosis. Science. 1999;284(5412):336-339.

46. Ditunno JF Jr, Formal CS. Chronic spinal cord injury. N Engl J Med. 1994;330(8):550-556.

47. Cao QL, Howard RM, Dennison JB, Whittemore SR Differentiation of engrafted neuronal-restricted precursor cells is inhibited in the traumatically injured spinal cord. Exp Neurol. 2002;177(2):349-359.

48. Han SS, Kang DY, Mujtaba T, Rao MS, Fischer I. Grafted lineage-restricted precursors differentiate exclusively into neurons in the adult spinal cord. Exp Neurol. 2002;177(2):360-375.

49. Niwa H, Yamamura K, Miyazaki J. Efficient selection for high-expression transfectants with a novel eukaryotic vector. Gene. 1991;108(2):193-199.

50. Pronichev IV, Lenkov DN. Functional mapping of the motor cortex of the white mouse by a microstimulation method. Neurosci Behav Physiol. 1998;28(1):80-85. 